\title{
Identify New Wheat Genotypes Resistant to Leaf and Stem Rusts under Egyptian Conditions
}

\section{M.A. Hasan}

Wheat Dis. Res. Dept., Plant Pathol. Res. Inst., ARC, Giza, Egypt.

\begin{abstract}
Qne hundred twenty three wheat genotypes derived from the International Maize and Wheat Improvement Centre (CIMMYT) were evaluated against leaf rust (Puccinia triticina) and stem rust (Puccinia graminis f.sp. tritici) as well as grain yield (1000 kernel weight and spike weight) at Gemmeiza Research Station during 2012/13 and 2013/14 growing seasons. Forty six and fifty nine wheat genotypes out of one hundred twenty three were resistant to leaf and stem rust diseases during 2012/13 growing season. While forty eight and sixty seven genotypes showed resistant reactions to both leaf and stem rusts during 2013/14. Regarding to 1000 kernel weight (g), 23 and 20 genotypes showed high yield during 2012/13 and 2013/14 growing seasons, respectively. Wheat genotypes No. 8, 31, 39, 40, 72, 80,101 and 123 were resistant to leaf and stem rust diseases during the two successive seasons and showed high yield, these genotypes displayed various levels of adult plant resistance in the field and could be used as an important source for breeding high yielding resistant varieties.
\end{abstract}

Keywords: Leaf, resistance, stem rust, wheat and yield components.

Bread wheat (Triticum aestivum L.) is one of the most important field crops in Egypt. Nevertheless, the yield production is not sufficient to cover the local consumption in Egypt (Anonymous, 2013). Breeding high yield varieties resistant to leaf and stem rusts is a very important objective in Egypt. To fill the gap between the production and consumption, however yield of wheat can be increased by increasing the cultivated area (horizontally) or by increasing the yield per unit area (vertically). The only alternative method is to obtain higher yield per unit area by growing high yielding varieties resistant to diseases.

Rust diseases of wheat, i.e. leaf rust (Puccinia triticina Eriks.) and stem rust (Puccinia graminis Pers. f.sp. tritici Eriks. \& E. Henn.), are still the most dangerous biotic stress that threaten wheat production in Egypt and in several wheat growing areas of the world. This is mainly due to the appearance of aggressive races of the pathogen (Singh et al., 2005).

Leaf rust causes severe losses in grain yield which may reach more than $20 \%$ on the susceptible cultivars depending on environmental conditions, level of resistance, stage of crop development at the initial stage of infection and the dominant physiologic races (Nazim et al., 1983). While, wheat stem rust fungus could affect the entire wheat crop, especially during the early growth stages leading to the blocking of the vascular system hence stunting and lodging of weak stalks eventually causing yield losses of even $100 \%$ due to shrivelled grain and damaged 
tillers (Kokhmetova et al., 2011 and Boukhatem et al., 2002). In Egypt, yield losses due to stem rust ranged from $1.96 \%$ to $8.21 \%$ on the Egyptian wheat cultivars (Ashmawy et al., 2013). In most cases, susceptible wheat cultivars were replaced with new resistant one (Rattu et al., 2007). Meanwhile, various control options are available for combating wheat rusts using resistant varieties is the most effective and safety one.

This aim of this study was to identify new wheat genotypes resistant to leaf and stem rusts under Egyptian conditions. Also, to evaluate these genotypes for yield components and select lines that perform high yield and showing desirable resistance against rust diseases to be used in Egyptian breeding program.

\section{Materials and Methods}

One hundred twenty three genotypes were used in this study (Table 1), these genotypes were derived from (CIMMYT). This experiment was carried out at Gemmeiza Research Station during 2012/13 and 2013/14 growing seasons. Each tested wheat genotype was planted in two rows of $1 \mathrm{~m}$ length with 4 replicates. Normal agricultural wheat practices were applied and the plots were surrounded by spreader area planted with a mixture of highly susceptible wheat varieties, i.e. Triticum spelta sahariensis, Morocco, Thatcher and Max to spread rust inoculum. For field inoculation with leaf and stem rusts, the spreader plants were sprayed with a mist of water and dusted with mixture of urediniospores of the prevalent rust races mixed with talcum powder at a rate of 1 (spore): 20 (talcum powder). The inoculation of all plants was carried out at booting stage according to the method of Tervet and Cassel (1951).

\section{Disease assessment:}

Leaf and stem rust severities and reactions were recorded for each genotype using the modified Cobb's scale (Peterson et al., 1948). Area under disease progress curve (AUDPC) was assessed for each genotype according to the equation adopted by Stubbs et al. (1986).

$$
\mathrm{AUDPC}=\mathrm{D}[1 / 2(\mathrm{Y} 1+\mathrm{Yk})+(\mathrm{Y} 2+\mathrm{Y} 3+----+\mathrm{Yk}-1)]
$$

Whereas: $\mathrm{D}=$ days between two consecutive recording (time intervals).

$\mathrm{Y} 1+\mathrm{Yk}=$ Sum of the first and last scores.

$\mathrm{Y} 2+\mathrm{Y} 3+--\cdot-+$ Yk-1= Sum of all in between disease scores.

Coefficient of infection (CI) was calculated by multiplying rust severity with constant values of infection type (IT). The constant values for infection types were used based on; $\mathrm{R}=0.2, \mathrm{MR}=0.4, \mathrm{MS}=0.8$ and $\mathrm{S}=1$ (Stubbs et al., 1986). Average coefficient of infection (ACI) was derived from the sum of CI values of each line divided by the number of locations.

Yield components:

Thousand kernels weigh ( $\mathrm{g}$ ) and spike weight were studied for each genotype, thousand kernels weight was measured by threshing the kernels from the spikes and 1000 seed from each genotype were calculated and weighted. 
Table 1. List of the tested wheat genotypes, cross name and origin

\begin{tabular}{|c|c|c|}
\hline No. & Designation & Origin \\
\hline 1 & PBW343 & MXI10-11\M6SRRSNI210 \\
\hline 2 & KINGBIRD \#1 & MXI10-11\M6SRRSNI211 \\
\hline 3 & WBLL1 $* 2 /$ KURUKU//HEILO & MXI10-11\M6SRRSNI4 \\
\hline 4 & WBLL1*2/KURUKU//HEILO & MXI10-11\M6SRRSN15 \\
\hline 5 & ATTILA $* 2 / \mathrm{PBW} 65 * 2 / / \mathrm{KACHU}$ & MXI10-11\M6SRRSNI6 \\
\hline 6 & ATTILA $* 2 / \mathrm{PBW} 65 * 2 / / \mathrm{KACHU}$ & MXI10-11\M6SRRSNI7 \\
\hline 7 & $\begin{array}{l}\text { WBLL1*2/KUKUNA/5/PSN/BOW//SERI/3/MILAN/4/ATTILA/6/W } \\
\text { BLL1*2/KKTS }\end{array}$ & MXI10-11 \M6SRRSN18 \\
\hline 8 & CNO79//PF70354/MUS/3/PASTOR/4/BAV92*2/5/HAR311 & MXI10-11\M6SRRSN\9 \\
\hline 9 & CNO79//PF70354/MUS/3/PASTOR/4/BAV92*2/5/FH6-1-7 & MXI10-11\M6SRRSN\10 \\
\hline 10 & CNO79//PF70354/MUS/3/PASTOR/4/BAV92*2/5/FH6-1-7 & MXI10-11\M6SRRSN\11 \\
\hline 11 & CNO79//PF70354/MUS/3/PASTOR/4/BAV92*2/5/FH6-1-7 & MXI10-11\M6SRRSN\12 \\
\hline 12 & CNO79//PF70354/MUS/3/PASTOR/4/BAV92*2/5/FH6-1-7 & MXI10-11\M6SRRSN\13 \\
\hline 13 & CNO79//PF70354/MUS/3/PASTOR/4/BAV92*2/5/FH6-1-7 & MXI10-11\M6SRRSN\15 \\
\hline 14 & KACHU \#1/KIRITATI//KACHU & MXI10-11\M6SRRSN\16 \\
\hline 15 & SAUAL/YANAC//SAUAL & MXI10-11\M6SRRSN\17 \\
\hline 16 & SAUAL/YANAC//SAUAL & MXI10-11\M6SRRSN\19 \\
\hline 17 & PRL/2*PASTOR $* 2 / /$ FH6-1-7 & MXI10-11\M6SRRSN120 \\
\hline 18 & PBW343*2/KUKUNA*2//FRTL/PIFED & MXI10-11\M6SRRSN\21 \\
\hline 19 & PBW343*2/KUKUNA*2//FRTL/PIFED & MXI10-11\M6SRRSNI22 \\
\hline 20 & PBW343*2/KUKUNA*2//FRTL/PIFED & MXI10-11\M6SRRSNI24 \\
\hline 21 & UP2338*2/KKTS*2//YANAC & MXI10-11\M6SRRSN\27 \\
\hline 22 & UP2338*2/KKTS*2//YANAC & MXI10-11\M6SRRSNI28 \\
\hline 23 & BAV92//IRENA/KAUZ/3/HUITES*2/4/MURGA & MXI10-11\M6SRRSN\35 \\
\hline 24 & BAV92//IRENA/KAUZ/3/HUITES*2/4/MURGA & MXI10-11\M6SRRSNI36 \\
\hline 25 & $\begin{array}{l}\text { ROLF07*2/5/REH/HARE//2*BCN/3/CROC_1/AE.SQUARROSA } \\
(213) / / \text { PGO/4/HUITES }\end{array}$ & MXI10-11 \M6SRRSNI37 \\
\hline 26 & $\begin{array}{l}\text { BAV92//IRENA/KAUZ/3/HUITES*2/4/CROC_1/AE.SQUARROSA } \\
(224) / / \text { KULIN/3/WESTONIA }\end{array}$ & MXI10-11\M6SRRSN\40 \\
\hline 27 & $\begin{array}{l}\text { BAV92//IRENA/KAUZ/3/HUITES*2/4/CROC_1/AE.SQUARROSA } \\
\text { (224)//KULIN/3/WESTONIA }\end{array}$ & MXI10-11\M6SRRSN\41 \\
\hline 28 & $\begin{array}{l}\text { BAV92//IRENA/KAUZ/3/HUITES*2/4/CROC_1/AE.SQUARROSA } \\
\text { (224)//KULIN/3/WESTONIA }\end{array}$ & MXI10-11\M6SRRSNI42 \\
\hline 29 & ROLF07*2/5/FCT/3/GOV/AZ//MUS/4/DOVE/BUC & MXI10-11\M6SRRSN\43 \\
\hline 30 & WAXWING/KIRITATI*2/3/C80.1/3*BATAVIA//2*WBLL1 & MXI10-11\M6SRRSN\46 \\
\hline 31 & FRNCLN/ROLF07 & MXI10-11\M6SRRSNI47 \\
\hline 32 & $\begin{array}{l}\text { ALTAR84/AE.SQUARROSA(221)//3*BORL95/3/URES/JUN//KAU } \\
\text { Z/4/WBLL1/5/MILAN/S87230//BAV92 }\end{array}$ & MXI10-11 \M6SRRSNI50 \\
\hline 33 & $\begin{array}{l}\text { ALTAR84/AE.SQUARROSA(221)//3*BORL95/3/URES/JUN//KAU } \\
\text { Z/4/WBLL1/5/MILAN/S87230//BAV92 }\end{array}$ & MXI10-11\M6SRRSNI51 \\
\hline 34 & FRNCLN/TECUE \#1 & MXI10-11\M6SRRSNI52 \\
\hline 35 & TRCH/HUIRIVIS \#1 & MXI10-11\M6SRRSNI53 \\
\hline 36 & TRCH/KBIRD & MXI10-11\M6SRRSNI54 \\
\hline 37 & BECARD/AKURI & MXI10-11\M6SRRSNI56 \\
\hline 38 & WAXWING/4/SNI/TRAP\#1/3/KAUZ*2/TRAP//KAUZ/5/AKURI & MXI10-11\M6SRRSN\60 \\
\hline 39 & KINGBIRD \#1//INQALAB 91*2/TUKURU & MXI10-11\M6SRRSN|61 \\
\hline 40 & KINGBIRD \#1//INQALAB 91*2/TUKURU & MXI10-11\M6SRRSN\62 \\
\hline 41 & PBW343*2/KUKUNA//TECUE \#1 & MXI10-11\M6SRRSN\63 \\
\hline 42 & PBW343*2/KUKUNA//TECUE \#1 & MXI10-11\M6SRRSNI64 \\
\hline 43 & $\begin{array}{l}\text { BL2064//SW89-5124*2/FASAN/3/TILHI/5/KAUZ//ALTAR } \\
\text { 84/AOS/3/KAUZ/4/SW94.15464 }\end{array}$ & MXI10-11 \M6SRRSN\66 \\
\hline 44 & $\begin{array}{l}\text { ROLF07*2/5/REH/HARE//2*BCN/3/CROC_1/AE.SQUARROSA } \\
(213) / / \text { PGO/4/HUITES }\end{array}$ & MXI10-11\M6SRRSNI68 \\
\hline
\end{tabular}


Table 1: Continued

\begin{tabular}{|c|c|c|}
\hline 45 & MUU \#1//PBW343*2/KUKUNA/3/MUU & MXI10-11\M6SRRSNI73 \\
\hline 46 & $\begin{array}{l}\text { WAXWING/4/BL 1496/MILAN/3/CROC_1/AE.SQUARROSA } \\
(205) / / \text { KAUZ/5/FRNCLN }\end{array}$ & MXI10-11 \M6SRRSNI75 \\
\hline 47 & UP2338*2/VIVITSI/3/FRET2/TUKURU//FRET2/4/MISR 1 & MXI10-11\M6SRRSNI76 \\
\hline 48 & WAXWING*2/HEILO & MXI10-11 \M6SRRSNI82 \\
\hline 49 & KIRITATI/4/2*BAV92//IRENA/KAUZ/3/HUITES & MXI10-11\M6SRRSNI86 \\
\hline 50 & KIRITATI/4/2*BAV92//IRENA/KAUZ/3/HUITES & MXI10-11\M6SRRSNI87 \\
\hline 51 & $\begin{array}{l}\text { OASIS/SKAUZ//4*BCN/3/2*PASTOR/5/FRET2 } 2 \text { 2/4/SNI/TRAP\#1/3/ } \\
\text { KAUZ*2/TRAP//KAUZ/6/SAUAL \#1 }\end{array}$ & MXI10-11\M6SRRSNI89 \\
\hline 52 & KZA//WH 542/2*PASTOR/3/BACEU \#1 & MXI10-11\M6SRRSNI90 \\
\hline 53 & $\mathrm{KFA} / 2 * \mathrm{KACHU}$ & MXI10-11\M6SRRSN\93 \\
\hline 54 & $\mathrm{KFA} / 2 * \mathrm{KACHU}$ & MXI10-11\M6SRRSNI94 \\
\hline 55 & $\mathrm{KFA} / 2 * \mathrm{KACHU}$ & MXI10-11\M6SRRSN\95 \\
\hline 56 & $\begin{array}{l}\text { FRET2*2/4/SNI/TRAP\#1/3/KAUZ*2/TRAP//KAUZ*2/5/BOW/URE } \\
\text { S//2*WEAVER/3/CROC_1/AE.SQUARROSA }(213) / / \text { PGO }\end{array}$ & MXI10-11 \M6SRRSN\97 \\
\hline 57 & $\begin{array}{l}\text { FRET2*2/4/SNI/TRAP\#1/3/KAUZ*2/TRAP//KAUZ*2/5/BOW/URE } \\
\text { S//2*WEAVER/3/CROC_1/AE.SQUARROSA }(213) / / \text { PGO }\end{array}$ & MXI10-11 \M6SRRSNI98 \\
\hline 58 & WBLL1/MUU \#1 & MXI10-11\M6SRRSN\101 \\
\hline 59 & ATTILA*2/PBW65//MURGA & MXI10-11\M6SRRSN\105 \\
\hline 60 & $\begin{array}{l}\text { BAV92//IRENA/KAUZ/3/HUITES/6/ALD/CEP75630//CEP75234/PT } \\
\text { 7219/3/BUC/BJY/4/CBRD/5/TNMU/PF85487 }\end{array}$ & MXI10-11\M6SRRSN\106 \\
\hline 61 & WBLL1*2/KURUKU//HEILO & MXI10-11\M6SRRSN $\backslash 108$ \\
\hline 62 & $\begin{array}{l}\text { ROLF07*2/4/CROC_1/AE.SQUARROSA } \\
(205) / / \text { BORL95/3/2*MILAN }\end{array}$ & MXI10-11\M6SRRSN $\backslash 109$ \\
\hline 63 & $\begin{array}{l}\text { ROLF07*2/4/CROC_1/AE.SQUARROSA } \\
(205) / / \text { BORL95/3/2*MILAN }\end{array}$ & MXI10-11\M6SRRSN $\backslash 110$ \\
\hline 64 & $\begin{array}{l}\text { WBLL1 } * 2 / \text { KUKUNA/5/PSN/BOW//SERI/3/MILAN/4/ATTILA/6/W } \\
\text { BLL1 } * 2 / \text { KKTS }\end{array}$ & MXI10-11\M6SRRSN\113 \\
\hline 65 & WAXWING/KIRITATI*2//YANAC & MXI10-11\M6SRRSN\115 \\
\hline 66 & BAV92//IRENA/KAUZ/3/HUITES*2/4/PVN & MXI10-11\M6SRRSN $\backslash 116$ \\
\hline 67 & CNO79//PF70354/MUS/3/PASTOR/4/BAV92*2/5/HAR311 & MXI10-11\M6SRRSN\117 \\
\hline 68 & CNO79//PF70354/MUS/3/PASTOR/4/BAV92*2/5/HAR311 & MXI10-11\M6SRRSN\118 \\
\hline 69 & CNO79//PF70354/MUS/3/PASTOR/4/BAV92*2/5/HAR311 & MXI10-11\M6SRRSN\119 \\
\hline 70 & CNO79//PF70354/MUS/3/PASTOR/4/BAV92*2/5/HAR311 & MXI10-11\M6SRRSN\124 \\
\hline 71 & CNO79//PF70354/MUS/3/PASTOR/4/BAV92*2/5/HAR311 & MXI10-11\M6SRRSN\126 \\
\hline 72 & $\begin{array}{l}\text { TACUPETO 2001/6/CNDO/R143//ENTE/MEXI_2/3/AEGILOPS } \\
\text { SQUARROSA (TAUS)/4/WEAVER/5/PASTOR/7/ROLF07 }\end{array}$ & MXI10-11\M6SRRSN\128 \\
\hline 73 & ROLF07 $* 2 / D I A M O N D B I R D$ & MXI10-11\M6SRRSN\129 \\
\hline 74 & SAUAL/YANAC//SAUAL & MXI10-11\M6SRRSN\130 \\
\hline 75 & SAUAL/YANAC//SAUAL & MXI10-11\M6SRRSN\131 \\
\hline 76 & SAUAL/YANAC//SAUAL & MXI10-11\M6SRRSN\132 \\
\hline 77 & PRL/2*PASTOR $* 2 / / F H 6-1-7$ & MXI10-11\M6SRRSN\133 \\
\hline 78 & $\begin{array}{l}\text { CS/TH.SC//3*PVN/3/MIRLO/BUC/4/URES/JUN//KAUZ/5/HUITES/ } \\
\text { 6/YANAC/7/CS/TH.SC//3*PVN/3/MIRLO/BUC/4/MILAN/5/TILHI }\end{array}$ & MXI10-11\M6SRRSN $\backslash 134$ \\
\hline 79 & $\begin{array}{l}\text { KAUZ/PASTOR//PBW343/3/HAR311/5/OASIS/SKAUZ//4*BCN/3/P } \\
\text { ASTOR/4/KAUZ*2/YACO//KAUZ }\end{array}$ & MXI10-11 \M6SRRSN $\backslash 137$ \\
\hline 80 & $\begin{array}{l}\text { KAUZ/PASTOR//PBW343/3/HAR311/5/OASIS/SKAUZ//4*BCN/3/P } \\
\text { ASTOR/4/KAUZ*2/YACO//KAUZ }\end{array}$ & MXI10-11\M6SRRSN $\backslash 138$ \\
\hline 81 & INQALAB $91 * 2 / \mathrm{KUKUNA} * 2 / / \mathrm{PVN}$ & MXI10-11\M6SRRSN\139 \\
\hline 82 & UP2338*2/KKTS $* 2 / / Y A N A C$ & MXI10-11\M6SRRSN\140 \\
\hline 83 & UP2338*2/KKTS*2//YANAC & MXI10-11【M6SRRSN\141 \\
\hline 84 & ROLF07*2/KACHU \#1 & MXI10-11\M6SRRSN\142 \\
\hline 85 & ATTILA*2/PBW65*2//MURGA & MXI10-11\M6SRRSN\143 \\
\hline 86 & $\begin{array}{l}\text { BAV92//IRENA/KAUZ/3/HUITES*2/4/CROC_1/AE.SQUARROSA } \\
(224) / / \text { KULIN/3/WESTONIA }\end{array}$ & MXI10-11\M6SRRSN\146 \\
\hline
\end{tabular}

Egypt. J. Phytopathol., Vol. 42, No. 2 (2014) 
Table 1: Continued

\begin{tabular}{|c|c|c|}
\hline 87 & $\begin{array}{l}\text { PFAU/WEAVER*2//BRAMBLING/3/KAUZ//TRAP\#1/BOW/4/PFA } \\
\text { U/WEAVER*2//BRAMBLING }\end{array}$ & MXI10-11 \M6SRRSN $\backslash 150$ \\
\hline 88 & FRNCLN/BECARD & MXI10-11\M6SRRSN\156 \\
\hline 89 & PARUS/FRANCOLIN \#1 & MXI10-11\M6SRRSN \157 \\
\hline 90 & SAUAL \#1/KACHU & MXI10-11\M6SRRSN\158 \\
\hline 91 & BLL1 $* 2 /$ BRAMBLING//KACHU & MXI10-11\M6SRRSN\159 \\
\hline 92 & MILAN/S87230//BAV92/3/AKURI \#1 & MXI10-11\M6SRRSN\163 \\
\hline 93 & QUAIU/TECUE \#1 & MXI10-11\M6SRRSN\165 \\
\hline 94 & ROLF07/KINGBIRD \#1 & MXI10-11\M6SRRSN\166 \\
\hline 95 & WBLL1 $* 2 /$ TUKURU//CROSBILL \# 1 & MXI10-11\M6SRRSN\168 \\
\hline 96 & WBLL1*2/TUKURU//CROSBILL \#1 & MXI10-11\M6SRRSN\169 \\
\hline 97 & KINGBIRD \#1/KACHU & MXI10-11\M6SRRSN\173 \\
\hline 98 & WAXWING/4/SNI/TRAP\#1/3/KAUZ $* 2 / T R A$ & MXI10-11\M6SRRSN\174 \\
\hline 99 & KINGBIRD \#1//INQALAB 91*2/TUKURU & MXI10-11\M6SRRSN\175 \\
\hline 100 & PBW343*2/KUKUNA//TECUE \# 1 & MXI10-11\M6SRRSN\177 \\
\hline 101 & PBW343*2/KUKUNA//TECUE \#1 & MXI10-11\M6SRRSN\178 \\
\hline 102 & JE\#1 & N179 \\
\hline 103 & $\begin{array}{l}\text { PVN//CAR422/ANA/5/BOW/CROW//BUC/PVN/3/YR/4/TRAP\#1/6/ } \\
\text { AKURI\#1 }\end{array}$ & MXI10-11\M6SRRSN $\backslash 182$ \\
\hline 104 & $\begin{array}{l}\text { BL2064//SW89-5124*2/FASAN/3/TILHI/5/KAUZ//ALTAR } \\
\text { 84/AOS/3/KAUZ/4/SW94.15464 }\end{array}$ & MXI10-11\M6SRRSN\184 \\
\hline 105 & $\begin{array}{l}\text { PBW343*2/KHVAKI/5/KA } \\
\text { 84/AOS/3/KAUZ/4/SW94.1 } \\
\end{array}$ & MXI10-11\M6SRRSN $\backslash 185$ \\
\hline 106 & TAM200/PASTOR//TOBA97/3/WHEAR & MXI10-11\M6SRRSN\186 \\
\hline 107 & $80.1 / 3 * \mathrm{BATAVIA} / / 2 * \mathrm{WBLL} 1 / 3 / 2 *$ FRET2/TUKURU//FRET2 & MXI10- \\
\hline 108 & $\begin{array}{l}\text { NAC/TH.AC//3*PVN/3/MIRLO/BUC/4/2*PASTOR/5/KACHU/6/KA } \\
\text { CHU }\end{array}$ & MXI10-11\M6SRRSN $\backslash 189$ \\
\hline 109 & $\begin{array}{l}\text { SITE/MO//PASTOR/3/TILHI/4/WAXWING/KIRITATI/5/PBW343*2 } \\
\text { /TUKURU }\end{array}$ & MXI10-11\M6SRRSN $\backslash 190$ \\
\hline 110 & $\begin{array}{l}\text { UP2338*2/4/SNI/TRAP\#1/3/KAUZ*2/TRAP//KAUZ/5/2*WAXWIN } \\
\text { G/4/SNI/TRAP\#1/3/KAUZ*2/TRAP//KAUZ }\end{array}$ & MXI10-11\M6SRRSN $\backslash 192$ \\
\hline 111 & $\begin{array}{l}\text { BAV92//IRENA/KAUZ/3/HUITES/4/GONDO/TNMU/5/BAV92//IR } \\
\text { ENA/KAUZ/3/HUITES }\end{array}$ & MXI10-11\M6SRRSN\195 \\
\hline 112 & J//HEILO/3/WBLL1*2/KURUKU & M6SRRSN $\backslash 196$ \\
\hline 113 & & N1197 \\
\hline 114 & KIRITATI/4/2*BAV92//IRENA/KAUZ/3/HUITES & MXI10-11\M6SR \\
\hline 115 & $\begin{array}{l}\text { OASIS/SKAUZ//4*BCN/3/2*PASTOR/5/FRET } 2 * 2 / 4 / \mathrm{SNI} / \mathrm{TRAP} \# 1 / 3 / \\
\text { KAUZ*2/TRAP//KAUZ/6/SAUAL \#1 }\end{array}$ & MXI10-11\M6SRRSNI201 \\
\hline 116 & SKAUZ/BAV92//2*WBLL1*2/KKTS & M6SRRSN 1202 \\
\hline 117 & KENYA NYANGUMI//2*ATTILA*2/PB & MXI10-11 \M6SRRSN\203 \\
\hline 118 & $\begin{array}{l}\text { TACUPETO 2001/6/CNDO/R143//ENTE/MEXI_2/3/AEGILOPS } \\
\text { SQUARROSA (TAUS)/4/WEAVER/5/PASTOR/7/ROLF07 }\end{array}$ & MXI10-11\M6SRRSN1204 \\
\hline 119 & $\begin{array}{l}\text { TACUPETO 2001/6/CNDO/R143//ENTE/MEXI_2/3/AEGILOPS } \\
\text { SQUARROSA (TAUS)/4/WEAVER/5/PASTOR/7/ROLF07 }\end{array}$ & MXI10-11\M6SRRSN1207 \\
\hline 120 & FRANCOLIN \#1/KIRITATI & MXI10-11\M6SRRSNI208 \\
\hline 121 & KENYA NYANGUMI/3/2*KAUZ/PASTOR//PBW343 & MXI10-11\M6SRRSNI209 \\
\hline 122 & PBW343*2/KUKUNA//TECUE \# 1 & MXI10-11\M6SRRSN\65 \\
\hline 123 & KSW/SAUAL//SAUAL & MXI10-11\M6SRRSN\91 \\
\hline
\end{tabular}

\section{Results}

A total of 123 wheat genotypes were tested for adult plant resistance to leaf and stem rust diseases as well as yield components (Tables 2, 3 and 4). 
Evaluation of wheat genotypes against leaf rust under field conditions:

Season 2012/13:

Data presented in Table (2) show that the leaf rust severity of the tested genotypes varied from 0 to $80 \%$ with different infection types under field conditions. Out of 123 tested genotypes, 46 genotypes showed desirable/acceptable resistance to leaf rust, whereas rust severities ranged from 0 to $10 \mathrm{R}$. Area under disease progress curve (AUDPC) and Coefficient of infection (CI) are in parallel line with rust severity, which gave values ranged from 0 to 80.5 (AUDPC) and from 0 to 2 (CI). High rust severity was scored on 32 genotypes during 2012/13 season with values ranged from $40-80 \%$. The rest wheat genotypes were in between.

Season 2013/14:

Data in Table (2) prove that number of resistant genotypes was decreased to thirty eight which gave resistant reaction ranged from 0 to 10R. On the other hand, eighty five genotypes showed different infection types (MR, MS and S) with different disease severity values ranged from 5 to $80 \%$. The highest values of Area under disease progress curve (AUDPC) and Coefficient of infection (CI) were observed on sixteen genotypes.

During the two successive seasons, twenty three genotypes, i.e. 1, 2, 8, 9, 10, 20, $21,29,31,34,39,40,65,72,73,74,80,81,82,91,101,115$ and 123, showed high levels of adult plant resistant to leaf rust which gave low rust severity (0-10R), low area under disease progress curve (0-80.5) and low coefficient of infection (0-2).

Regarding to leaf rust severity and infection types during the two seasons, out of the total entries tested, $39.02 \%$ were resistant, $6.09 \%$ were moderately resistant, $17 \%$ were moderately susceptible and the remaining $(37.39 \%)$ were completely susceptible to the disease (Fig.1).

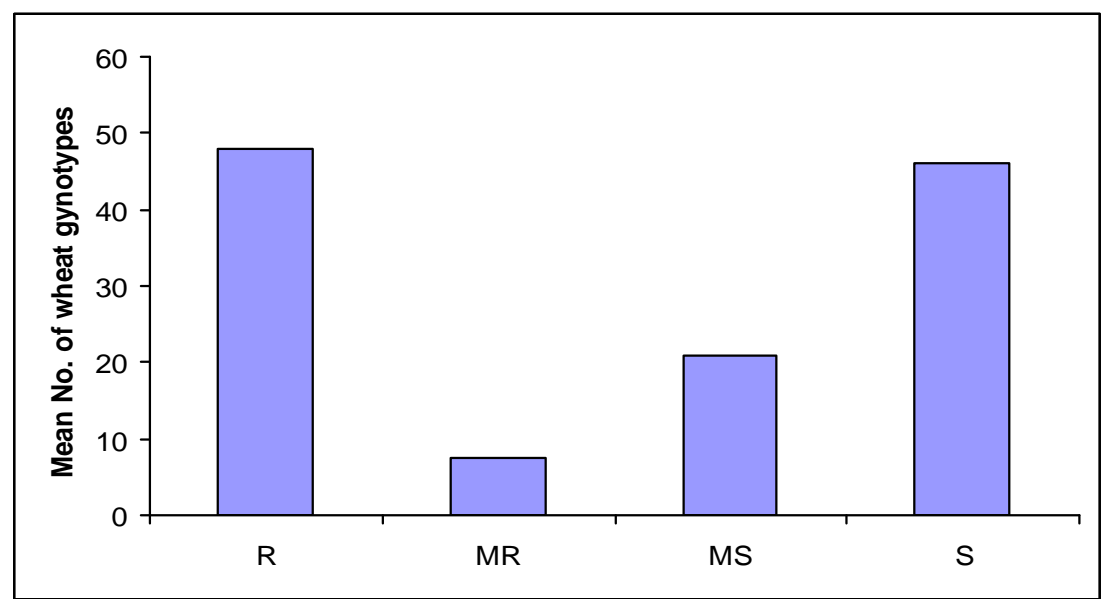

Fig. 1. Mean number of wheat genotypes distributed leaf rust infection types during the two seasons. Whereas: $S=$ susceptible, MS= moderately susceptible, $M R=$ moderately resistant and $R=$ resistant. 
IDENTIFY NEW WHEAT GENOTYPES RESISTANT TO ...

Table 2. Area under disease progress curve (AUDPC) and Coefficient of Infection of the tested wheat genotypes during 2012/13 and 2013/14 growing seasons

\begin{tabular}{|c|c|c|c|c|c|c|c|c|c|c|c|c|}
\hline \multirow{3}{*}{ No. } & \multicolumn{6}{|c|}{$2012 / 13$} & \multicolumn{6}{|c|}{ 2013/14 } \\
\hline & \multicolumn{3}{|c|}{ Leaf rust } & \multicolumn{3}{|c|}{ stem rust } & \multicolumn{3}{|c|}{ Leaf rust } & \multicolumn{3}{|c|}{ stem rust } \\
\hline & R.S.* & AUDPC & $\mathrm{ACI}$ & R.S. & AUDPC & $\mathrm{ACl}$ & R.S. & AUDPC & $\mathrm{ACI}$ & R.S. & AUDPC & $\mathrm{ACI}$ \\
\hline 1 & 0 & 0 & 0 & 0 & 0 & 0 & 0 & 0 & 0 & 0 & 0 & 0 \\
\hline 2 & 0 & 0 & 0 & 0 & 0 & 0 & 0 & 0 & 0 & 0 & 0 & 0 \\
\hline 3 & $60 \mathrm{~S}$ & 665 & 60 & $\operatorname{Tr} \mathrm{S}$ & 15 & 3 & $40 \mathrm{~S}$ & 525 & 40 & $5 \mathrm{Ms}$ & 55 & 4 \\
\hline 4 & 0 & 0 & 0 & $30 \mathrm{~S}$ & 265 & 30 & 0 & 0 & 0 & $10 \mathrm{Ms}$ & 91 & 8 \\
\hline 5 & $10 \mathrm{Mr}$ & 80.5 & 4 & 0 & 0 & 0 & $10 \mathrm{Mr}$ & 91 & 4 & TrR & 15 & 0.6 \\
\hline 6 & $30 \mathrm{Mr}$ & 300 & 12 & $\operatorname{TrMs}$ & 15 & 2.4 & $5 \mathrm{R}$ & 55 & 1 & $5 \mathrm{Ms}$ & 55 & 4 \\
\hline 7 & 0 & 0 & 0 & 0 & 0 & 0 & $10 \mathrm{~S}$ & 91 & 10 & $5 \mathrm{R}$ & 55 & 1 \\
\hline 8 & 0 & 0 & 0 & 0 & 0 & 0 & 0 & 0 & 0 & 0 & 0 & 0 \\
\hline 9 & 0 & 0 & 0 & 0 & 0 & 0 & $10 \mathrm{~S}$ & 155 & 10 & 0 & 0 & 0 \\
\hline 10 & $30 \mathrm{~S}$ & 415 & 30 & 0 & 0 & 0 & $10 \mathrm{~S}$ & 165 & 10 & 0 & 0 & 0 \\
\hline 11 & $20 \mathrm{Mr}$ & 155 & 8 & $10 \mathrm{~S}$ & 80.5 & 10 & $40 \mathrm{~S}$ & 248 & 40 & 0 & 0 & 0 \\
\hline 12 & $60 \mathrm{Ms}$ & 665 & 48 & $30 \mathrm{~S}$ & 300 & 30 & Trs & 15 & 3 & TrR & 15 & 0.6 \\
\hline 13 & TrR & 15 & 0.6 & $\operatorname{Tr} \mathrm{S}$ & 15 & 3 & $20 \mathrm{~S}$ & 155 & 20 & $5 \mathrm{R}$ & 55 & 1 \\
\hline 14 & $20 \mathrm{Ms}$ & 155 & 16 & $\operatorname{Tr} \mathrm{S}$ & 15 & 3 & $30 \mathrm{~S}$ & 265 & 30 & 0 & 0 & 0 \\
\hline 15 & $20 \mathrm{Ms}$ & 155 & 16 & 0 & 0 & 0 & 0 & 0 & 0 & 0 & 0 & 0 \\
\hline 16 & 0 & 0 & 0 & $10 \mathrm{R}$ & 91 & 2 & $20 \mathrm{R}$ & 155 & 4 & $10 \mathrm{Ms}$ & 91 & 8 \\
\hline 17 & 0 & 0 & 0 & $10 \mathrm{~S}$ & 91 & 10 & 0 & 0 & 0 & $20 \mathrm{~S}$ & 168 & 20 \\
\hline 18 & $10 \mathrm{Mr}$ & 98 & 4 & 0 & 0 & 0 & $5 \mathrm{Ms}$ & 55 & 4 & TrMs & 15 & 2.4 \\
\hline 19 & $10 \mathrm{Mr}$ & 98 & 4 & $40 \mathrm{~S}$ & 322 & 40 & $30 \mathrm{Ms}$ & 300 & 24 & 0 & 0 & 0 \\
\hline 20 & 0 & 0 & 0 & 0 & 0 & 0 & 0 & 0 & 0 & $5 \mathrm{~S}$ & 55 & 5 \\
\hline 21 & 0 & 0 & 0 & 0 & 0 & 0 & 0 & 0 & 0 & $5 \mathrm{~S}$ & 55 & 5 \\
\hline 22 & $20 \mathrm{Ms}$ & 157 & 16 & $20 \mathrm{~S}$ & 155 & 20 & $10 \mathrm{~S}$ & 165 & 10 & $\operatorname{TrS}$ & 15 & 3 \\
\hline 23 & $10 \mathrm{R}$ & 80.5 & 2 & 0 & 0 & 0 & $10 \mathrm{R}$ & 80.5 & 2 & 0 & 0 & 0 \\
\hline 24 & $10 \mathrm{~S}$ & 165 & 10 & $5 \mathrm{~S}$ & 55 & 5 & $5 \mathrm{R}$ & 55 & 1 & 0 & 0 & 0 \\
\hline 25 & $20 \mathrm{Ms}$ & 157 & 16 & 0 & 0 & 0 & $40 \mathrm{~S}$ & 525 & 40 & $5 \mathrm{~S}$ & 55 & 5 \\
\hline 26 & $10 \mathrm{Mr}$ & 130 & 4 & $10 \mathrm{~S}$ & 130 & 10 & $10 \mathrm{Ms}$ & 98 & 8 & 0 & 0 & 0 \\
\hline 27 & $30 \mathrm{~S}$ & 4 & 30 & Trs & 15 & 3 & $60 \mathrm{~S}$ & 825 & 60 & 0 & 0 & 0 \\
\hline 28 & $60 \mathrm{~S}$ & 82 & 60 & Trs & 15 & 3 & $60 \mathrm{~S}$ & 825 & 60 & $\operatorname{TrS}$ & 15 & 3 \\
\hline 29 & $80 \mathrm{~S}$ & & 80 & 0 & 0 & 0 & $40 \mathrm{~S}$ & 515 & 50 & 0 & 0 & 0 \\
\hline 30 & $80 \mathrm{~S}$ & 915 & 80 & 0 & 0 & 0 & TrS & 15 & 3 & $40 \mathrm{~S}$ & 525 & 40 \\
\hline 31 & 0 & 0 & 0 & 0 & 0 & 0 & 0 & 0 & 0 & 0 & 0 & 0 \\
\hline 32 & 0 & 0 & 0 & $30 \mathrm{~S}$ & 300 & 30 & 0 & 0 & 0 & $5 \mathrm{~S}$ & 55 & 5 \\
\hline 33 & $80 \mathrm{~S}$ & 925 & 80 & $5 \mathrm{~S}$ & 55 & 5 & $40 \mathrm{~S}$ & 322 & 40 & $40 \mathrm{~S}$ & 322 & 40 \\
\hline 34 & 0 & 0 & 0 & 0 & 0 & 0 & 0 & 0 & 0 & 0 & 0 & 0 \\
\hline 35 & $30 \mathrm{Ms}$ & & 24 & Trs & 15 & 3 & $10 \mathrm{R}$ & 91 & 2 & 0 & 0 & 0 \\
\hline 36 & $80 \mathrm{~S}$ & 125 & 80 & 0 & 0 & 0 & $5 \mathrm{Ms}$ & 55 & 4 & 0 & 0 & 0 \\
\hline 37 & $80 \mathrm{~S}$ & 125 & 80 & TrMs & 15 & 2.8 & $\operatorname{TrR}$ & 15 & 0.6 & 0 & 0 & 0 \\
\hline 38 & $80 \mathrm{~S}$ & 1125 & 80 & $10 \mathrm{Mr}$ & 91 & 4 & 0 & 0 & 0 & 0 & 0 & 0 \\
\hline 39 & 0 & 0 & 0 & 0 & 0 & 0 & 0 & 0 & 0 & 0 & 0 & 0 \\
\hline 40 & 0 & 0 & 0 & 0 & 0 & 0 & 0 & 0 & 0 & 0 & 0 & 0 \\
\hline 41 & $60 \mathrm{~S}$ & 825 & 60 & $10 \mathrm{R}$ & 80.5 & 2 & $40 \mathrm{Ms}$ & 248 & 32 & 0 & 0 & 0 \\
\hline 42 & $10 \mathrm{Ms}$ & 91 & 8 & 0 & 0 & 0 & $\mathrm{TrMs}$ & 15 & 2.4 & 0 & 0 & 0 \\
\hline 43 & $40 \mathrm{~S}$ & 525 & 40 & $10 \mathrm{R}$ & 91 & 2 & TrMs & 15 & 2.4 & $5 \mathrm{~S}$ & 55 & 5 \\
\hline 44 & $40 \mathrm{~S}$ & 525 & 40 & $10 \mathrm{~S}$ & 165 & 10 & 0 & 0 & 0 & $30 \mathrm{~S}$ & 300 & 30 \\
\hline 45 & $10 \mathrm{Mr}$ & 98 & 4 & $20 \mathrm{~S}$ & 168 & 20 & $30 \mathrm{Ms}$ & 300 & 24 & $40 \mathrm{~S}$ & 248 & 40 \\
\hline 46 & TrMs & 15 & 2.4 & 0 & 0 & 0 & $10 \mathrm{R}$ & 98 & 2 & 0 & 0 & 0 \\
\hline
\end{tabular}


Table 2: Continued

\begin{tabular}{|c|c|c|c|c|c|c|c|c|c|c|c|c|}
\hline 47 & 0 & 0 & 0 & $\operatorname{TrS}$ & 15 & 3 & 0 & 0 & 0 & $10 \mathrm{~S}$ & 98 & 10 \\
\hline 48 & $60 \mathrm{~S}$ & 825 & 60 & $10 \mathrm{Ms}$ & 98 & 8 & 0 & 0 & 0 & 0 & 0 & 0 \\
\hline 49 & $10 \mathrm{R}$ & 91 & 2 & 30Ms & 300 & 24 & $\operatorname{TrS}$ & 15 & 3 & $10 \mathrm{Ms}$ & 91 & 8 \\
\hline 50 & $10 \mathrm{R}$ & 91 & 2 & $40 \mathrm{~S}$ & 322 & 40 & $5 S$ & 55 & 5 & $80 \mathrm{~S}$ & 1125 & 80 \\
\hline 51 & 0 & 0 & 0 & $10 \mathrm{Mr}$ & 0 & 0 & $20 \mathrm{Ms}$ & 155 & 16 & $20 \mathrm{Mr}$ & 155 & 8 \\
\hline 52 & $30 \mathrm{~S}$ & 415 & 30 & 0 & 0 & 0 & \begin{tabular}{|l|}
0 \\
\end{tabular} & 0 & 0 & 0 & 0 & 0 \\
\hline 53 & $80 \mathrm{~S}$ & 925 & 80 & $20 \mathrm{~S}$ & 157 & 20 & 0 & 0 & 0 & 0 & 0 & 0 \\
\hline 54 & $80 \mathrm{~S}$ & 925 & 80 & Tr Mr & 15 & 1.2 & $\mathrm{TrS}$ & 15 & 3 & 0 & 0 & 0 \\
\hline 55 & $10 \mathrm{~S}$ & 130 & 10 & 0 & 0 & 0 & $20 \mathrm{Mr}$ & 157 & 8 & $10 \mathrm{Ms}$ & 130 & 8 \\
\hline 56 & 0 & 0 & 0 & 0 & 0 & 0 & $10 \mathrm{~S}$ & 165 & 10 & $\operatorname{TrS}$ & 55 & 3 \\
\hline 57 & 0 & 0 & 0 & 0 & 0 & 0 & $10 \mathrm{~S}$ & 165 & 10 & $10 \mathrm{R}$ & 130 & 2 \\
\hline 58 & $10 \mathrm{~S}$ & 165 & 10 & $10 \mathrm{Ms}$ & 91 & 8 & $30 \mathrm{Ms}$ & 280 & 24 & 0 & 0 & 0 \\
\hline 59 & 0 & 0 & 0 & $\mathrm{TrMr}$ & 15 & 1.2 & $10 \mathrm{Ms}$ & 91 & 8 & $40 \mathrm{~S}$ & 322 & 40 \\
\hline 60 & 0 & 0 & 0 & $30 \mathrm{~S}$ & 300 & 30 & $10 \mathrm{Ms}$ & 91 & 8 & $30 \mathrm{~S}$ & 415 & 30 \\
\hline 61 & $60 \mathrm{~S}$ & 825 & 60 & TrMs & 15 & 2.4 & TrS & 15 & 3 & $30 \mathrm{~S}$ & 415 & 30 \\
\hline 62 & $10 \mathrm{Ms}$ & 80.5 & 8 & TrMs & 15 & 2.4 & $10 \mathrm{Ms}$ & 91 & 8 & $\operatorname{TrS}$ & 15 & 3 \\
\hline 63 & TrMr & 15 & 1.2 & $30 \mathrm{~S}$ & 415 & 30 & 20R & 230 & 6 & TrMs & 15 & 2.4 \\
\hline 64 & 30Ms & 280 & 24 & 0 & 0 & 0 & 0 & 0 & 0 & $60 \mathrm{~S}$ & 925 & 60 \\
\hline 65 & 0 & 0 & 0 & 0 & 0 & 0 & 0 & 0 & 0 & 0 & 0 & 0 \\
\hline 66 & $10 \mathrm{~S}$ & 91 & 10 & $10 \mathrm{Ms}$ & 98 & 8 & 0 & 0 & 0 & $10 \mathrm{~S}$ & 157 & 10 \\
\hline 67 & $60 \mathrm{~S}$ & 925 & 60 & 0 & 0 & 0 & $10 \mathrm{Ms}$ & 91 & 8 & $30 \mathrm{~S}$ & 300 & 30 \\
\hline 68 & $10 \mathrm{R}$ & 91 & 2 & $10 \mathrm{~S}$ & 130 & 10 & TrMs & 15 & 2.4 & 0 & 0 & 0 \\
\hline 69 & $10 \mathrm{Ms}$ & 91 & 8 & $\operatorname{TrS}$ & 15 & 3 & TrMs & 15 & 2.4 & 0 & 0 & 0 \\
\hline 70 & $50 \mathrm{~S}$ & 425 & 50 & TrMs & 15 & 2.4 & $30 \mathrm{~S}$ & 415 & 30 & 0 & 0 & 0 \\
\hline 71 & 0 & 0 & 0 & 0 & 0 & 0 & $5 \mathrm{Ms}$ & 55 & 4 & 0 & 0 & 0 \\
\hline 72 & 0 & 0 & 0 & 0 & 0 & 0 & 0 & 0 & 0 & 0 & 0 & 0 \\
\hline 73 & $80 \mathrm{~S}$ & 925 & 80 & 0 & 0 & 0 & $30 \mathrm{~S}$ & 415 & 30 & 0 & 0 & 0 \\
\hline 74 & $80 \mathrm{~S}$ & 925 & 80 & 0 & 0 & 0 & $30 \mathrm{~S}$ & 385 & 30 & 0 & 0 & 0 \\
\hline 75 & 40Ms & 248 & 32 & $30 \mathrm{~S}$ & 280 & 30 & 0 & 0 & 0 & 0 & 0 & 0 \\
\hline 76 & $60 \mathrm{Ms}$ & 665 & 48 & $10 \mathrm{~S}$ & 98 & 10 & $30 \mathrm{Ms}$ & 248 & 24 & 0 & 0 & 0 \\
\hline 77 & 0 & 0 & 0 & 0 & 0 & 0 & $20 \mathrm{Mr}$ & 155 & 8 & $60 \mathrm{~S}$ & 825 & 60 \\
\hline 78 & $10 \mathrm{~S}$ & 130 & 10 & $20 \mathrm{Mr}$ & 155 & 8 & $30 \mathrm{~S}$ & 300 & 30 & $30 \mathrm{~S}$ & 280 & 30 \\
\hline 79 & $30 \mathrm{Ms}$ & 280 & 24 & $\operatorname{TrS}$ & 15 & 3 & 0 & 0 & 0 & 0 & 0 & 0 \\
\hline 80 & 0 & 0 & 0 & 0 & 0 & 0 & 0 & 0 & 0 & 0 & 0 & 0 \\
\hline 81 & $10 \mathrm{Ms}$ & 130 & 8 & 0 & 0 & 0 & $30 \mathrm{~S}$ & 415 & 30 & 0 & 0 & 0 \\
\hline 82 & 0 & 0 & 0 & 0 & 0 & 0 & 0 & 0 & 0 & 0 & 0 & 0 \\
\hline 83 & $60 \mathrm{~S}$ & 825 & 60 & $20 \mathrm{~S}$ & 168 & 20 & 0 & 0 & 0 & 0 & 0 & 0 \\
\hline 84 & $40 \mathrm{~S}$ & 322 & 40 & 0 & 0 & 0 & $5 \mathrm{~S}$ & 55 & 5 & $10 \mathrm{Ms}$ & 91 & 8 \\
\hline 85 & $10 \mathrm{Mr}$ & 80.5 & 4 & 0 & 0 & 0 & $20 \mathrm{Ms}$ & 157 & 16 & $40 \mathrm{~S}$ & 322 & 40 \\
\hline 86 & $\operatorname{TrS}$ & 15 & 3 & $20 \mathrm{Mr}$ & 155 & 8 & \begin{tabular}{l|}
0 \\
\end{tabular} & 0 & 0 & $10 \mathrm{~S}$ & 0 & 10 \\
\hline 87 & $30 \mathrm{~S}$ & 415 & 30 & $60 \mathrm{~S}$ & 665 & 60 & 0 & 0 & 0 & $\operatorname{TrS}$ & 0 & 3 \\
\hline 88 & $5 \mathrm{R}$ & 55 & 1 & 30Ms & 300 & 24 & $5 \mathrm{~S}$ & 55 & 5 & $30 \mathrm{~S}$ & 280 & 30 \\
\hline 89 & 0 & 0 & 0 & 0 & 0 & 0 & $40 \mathrm{Ms}$ & 248 & 32 & 0 & 0 & 0 \\
\hline 90 & $10 \mathrm{Ms}$ & 98 & 8 & $20 \mathrm{~S}$ & 168 & 20 & $10 \mathrm{Mr}$ & 130 & 4 & 0 & 0 & 0 \\
\hline 91 & 0 & 0 & 0 & 0 & 0 & 0 & \begin{tabular}{|l|}
0 \\
\end{tabular} & 0 & 0 & 0 & 0 & 0 \\
\hline 92 & 0 & 0 & 0 & $10 \mathrm{~S}$ & 130 & 10 & 0 & 0 & 0 & $20 \mathrm{~S}$ & 165 & 20 \\
\hline 93 & 0 & 0 & 0 & $5 \mathrm{R}$ & 55 & 1 & $20 \mathrm{~S}$ & 230 & 20 & $40 \mathrm{~S}$ & 525 & 40 \\
\hline 94 & $60 \mathrm{~S}$ & 825 & 60 & $\mathrm{TrS}$ & 15 & 3 & $10 \mathrm{~S}$ & 165 & 10 & $5 \mathrm{~S}$ & 55 & 5 \\
\hline 95 & $60 \mathrm{~S}$ & 665 & 60 & $30 \mathrm{~S}$ & 415 & 30 & $30 \mathrm{~S}$ & 300 & 30 & 0 & 0 & 0 \\
\hline 96 & $10 \mathrm{Ms}$ & 130 & 8 & 0 & 0 & 0 & $10 \mathrm{~S}$ & 165 & 10 & $5 \mathrm{~S}$ & 55 & 5 \\
\hline 97 & $20 \mathrm{Mr}$ & 155 & 8 & TrMs & 15 & 2.4 & $30 \mathrm{Mr}$ & 248 & 12 & $\operatorname{TrS}$ & 15 & 10 \\
\hline
\end{tabular}


Table 2: Continued

\begin{tabular}{|c|c|c|c|c|c|c|c|c|c|c|c|c|}
\hline 98 & $10 \mathrm{~S}$ & 130 & 10 & $5 \mathrm{~S}$ & 55 & 5 & 0 & 0 & 0 & 0 & 0 & 0 \\
\hline 99 & $20 \mathrm{Ms}$ & 157 & 16 & 0 & 0 & 0 & $5 \mathrm{~S}$ & 63 & 5 & 0 & 0 & 0 \\
\hline 100 & $40 \mathrm{~S}$ & 322 & 40 & 0 & 0 & 0 & 0 & 0 & 0 & 0 & 0 & 0 \\
\hline 101 & 0 & 0 & 0 & 0 & 0 & 0 & 0 & 0 & 0 & 0 & 0 & 0 \\
\hline 102 & 0 & 0 & 0 & $5 \mathrm{~S}$ & 55 & 5 & 0 & 0 & 0 & 0 & 0 & 0 \\
\hline 103 & TrMs & 15 & 2.4 & $20 \mathrm{~S}$ & 157 & 20 & $10 \mathrm{~S}$ & 130 & 0 & $30 \mathrm{Ms}$ & 280 & 24 \\
\hline 104 & $60 \mathrm{~S}$ & 825 & 60 & $20 \mathrm{Ms}$ & 155 & 16 & $60 \mathrm{Ms}$ & 665 & 48 & $20 \mathrm{~S}$ & 168 & 20 \\
\hline 105 & $30 \mathrm{~S}$ & 300 & 30 & 0 & 0 & 0 & 0 & 0 & 0 & 0 & 0 & 0 \\
\hline 106 & $70 \mathrm{~S}$ & 975 & 70 & $\mathrm{TrMs}$ & 15 & 2.4 & 0 & 0 & 0 & 0 & 0 & 0 \\
\hline 107 & $10 \mathrm{~S}$ & 165 & 10 & 0 & 0 & 0 & $\mathrm{TrS}$ & 15 & 3 & $50 \mathrm{Ms}$ & 875 & 40 \\
\hline 108 & 0 & 0 & 0 & $20 \mathrm{~S}$ & 168 & 20 & $40 \mathrm{~S}$ & 322 & 40 & $30 \mathrm{~S}$ & 280 & 30 \\
\hline 109 & 0 & 0 & 0 & $5 \mathrm{R}$ & 55 & 1 & $80 \mathrm{~S}$ & 925 & 80 & $80 \mathrm{~S}$ & 925 & 80 \\
\hline 110 & $40 \mathrm{~S}$ & 525 & 40 & $40 \mathrm{~S}$ & 322 & 40 & $40 \mathrm{~S}$ & 525 & 40 & $\mathrm{TrS}$ & 15 & 3 \\
\hline 111 & $\mathrm{TrMs}$ & 15 & 2.4 & $5 \mathrm{Ms}$ & 55 & 4 & $40 \mathrm{~S}$ & 525 & 40 & $10 \mathrm{~S}$ & 165 & 10 \\
\hline 112 & $10 \mathrm{~S}$ & 98 & 10 & 0 & 0 & 0 & $30 \mathrm{~S}$ & 300 & 30 & 0 & 0 & 0 \\
\hline 113 & $80 \mathrm{~S}$ & 1125 & 80 & $20 \mathrm{Mr}$ & 155 & 8 & $60 \mathrm{~S}$ & 825 & 60 & $30 \mathrm{Ms}$ & 280 & 24 \\
\hline 114 & 0 & 0 & 0 & $10 \mathrm{Mr}$ & 80.5 & 4 & 0 & 0 & 0 & 0 & 0 & 0 \\
\hline 115 & 0 & 0 & 0 & 0 & 0 & 0 & 0 & 0 & 0 & 0 & 0 & 0 \\
\hline 116 & $10 \mathrm{Ms}$ & 130 & 8 & $10 \mathrm{R}$ & 80.5 & 2 & $30 \mathrm{~S}$ & 415 & 30 & $30 \mathrm{~S}$ & 415 & 30 \\
\hline 117 & $80 \mathrm{~S}$ & 925 & 80 & $30 \mathrm{~S}$ & 415 & 30 & $30 \mathrm{~S}$ & 415 & 30 & $60 \mathrm{~S}$ & 925 & 60 \\
\hline 118 & $60 \mathrm{~S}$ & 815 & 80 & 0 & 0 & 0 & $10 \mathrm{~S}$ & 157 & 10 & 0 & 0 & 0 \\
\hline 119 & $10 \mathrm{~S}$ & 91 & 10 & $10 \mathrm{~S}$ & 168 & 10 & $10 \mathrm{~S}$ & 98 & 10 & $\mathrm{TrS}$ & 15 & 3 \\
\hline 120 & 0 & 0 & 0 & 0 & 0 & 0 & $40 \mathrm{~S}$ & 322 & 40 & $\mathrm{TrS}$ & 15 & 3 \\
\hline 121 & 0 & 0 & 0 & 0 & 0 & 0 & $10 \mathrm{Ms}$ & 80.5 & 8 & $10 \mathrm{~S}$ & 98 & 10 \\
\hline 122 & $40 \mathrm{~S}$ & 525 & 40 & $10 \mathrm{Ms}$ & 155 & 8 & 0 & 0 & 0 & 0 & 0 & 0 \\
\hline 123 & 0 & 0 & 0 & 0 & 0 & 0 & 0 & 0 & 0 & 0 & 0 & 0 \\
\hline
\end{tabular}

* R.S: Rust Severity, AUDPC: Area under Progress Curve and ACI: Average Coefficient of infections.

\section{Evaluation of wheat genotypes against stem rust under field conditions:}

\section{Season 2012/13:}

Data presented in Table (2) show that the stem rust severity of the tested genotypes varied from 0 to $60 \%$ during 2012/13. Sixty wheat genotypes were resistant and showed infection type from 0 to $\mathrm{R}$, whereas four genotypes, i.e. 19, 50, 87 and 110, showed high infection type ranged from 40S to 60S. Area under disease progress curve and coefficient of infection were in parallel line with rust severity.

Season 2013/14:

Data in Table (2) show that stem rust severity and disease incidence were low, as well as the highest numbers of resistant genotypes (67 genotypes) were observed during this season. High rust severity was scored on 12 genotypes ranged from 40-60\%. Also, data show that there are 34 genotypes gave high levels of adult plant resistant to stem rust during the two seasons, i.e. 1, 2, 8, 9, 10, 15, 23, 29, 31, 34, 36, $39,40,42,46,52,65,71,72,73,74,80,81,82,89,91,99,100,101,105,112,115$, 118 and 123 .

Stem rust disease pressure during the two seasons was low compared with leaf rust, and out of the total entries tested, $51.21 \%$ were resistant, $3.6 \%$ were moderately resistant, $10.9 \%$ were moderately susceptible and the remaining $(34.1 \%)$ were completely susceptible to the disease (Fig. 2). 


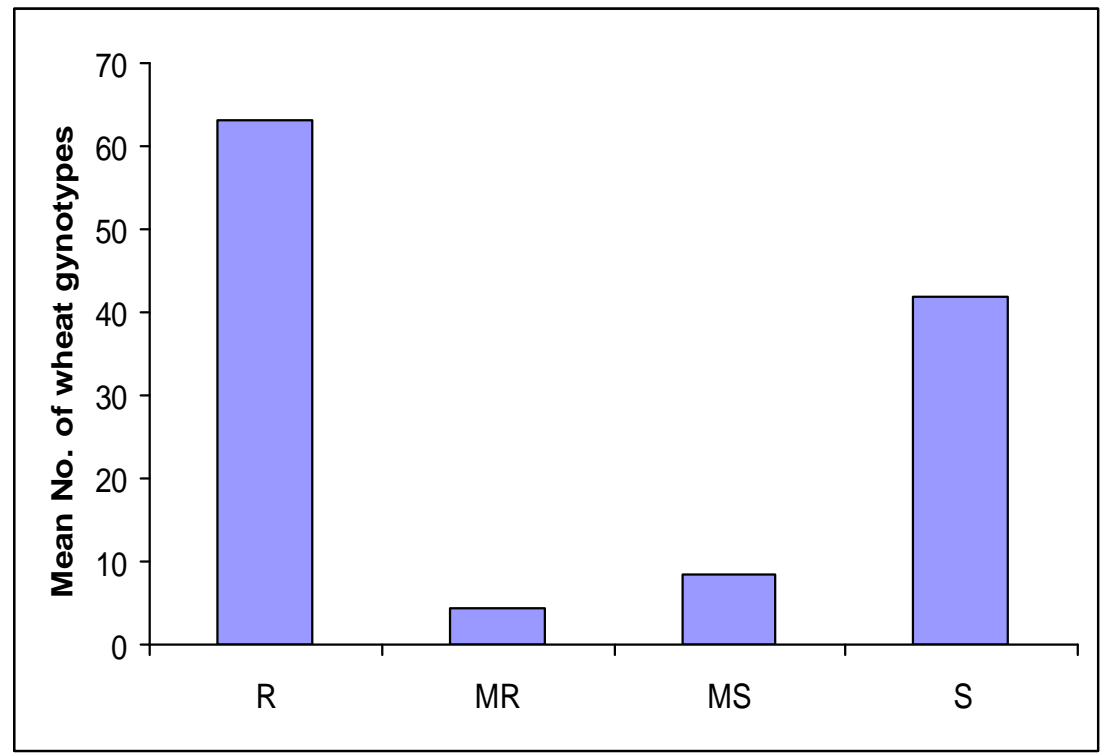

Fig. 2. Mean number of wheat genotypes distributed stem rust infection types during the two seasons. Whereas: $S=$ susceptible, $M S=$ moderately susceptible, $M R=$ moderately resistant and $R=$ resistant.

Yield components:

Season 2012/13:

Regarding 1000 kernels weigh and spike weight, data in Table (3) show that high values of 1000-kernel weight, were observed on 23 genotypes $(8,78,4,72,101,90$, $40,51,92,68,96,80,31,74,70,66,39,123,76,58,101,113$ and 17) which gave 1000 -kernel weigh ranged from (64.2-55.2g.). The rest wheat genotypes showed different 1000 -kernel weight from 34.00 to $55.00 \mathrm{~g}$. On the other hand, 7 wheat genotypes exhibited the highest values of spike weight $(\mathrm{g})$, these genotypes were, 57(5.64), 62(5.32), 13(4.9), 10(4.8), 106(4.84), 21(4.81) and 118(4.54).

Season 2013/14:

The obtained results in Table (3) reveal that 20 wheat genotypes showed the highest values of 1000-kernel weight, i.e. 43, 78, 90, 113, 92, 80, 74, 68, 70, 31, 39, $123,40,92,87,101,8,100,96$ and $17 \mathrm{~g}$, respectively. Eight wheat genotypes exhibited the highest values of spike weight (g), i.e. 62(5), 10(4.82), 63(4.8), 118(4.66), 23(4.62), 26(4.6), 106(4.61) and 52(4.5). 
IDENTIFY NEW WHEAT GENOTYPES RESISTANT TO ...

Table 3. 1000 kernel weight and Spike weight of the tested wheat genotypes during 2012/13 and 2013/14 growing seasons

\begin{tabular}{|c|c|c|c|c|}
\hline \multirow[b]{2}{*}{ No. } & \multicolumn{2}{|c|}{$2012 / 13$} & \multicolumn{2}{|c|}{$2013 / 14$} \\
\hline & Spike weight (g) & $\begin{array}{l}1000 \text { kernel } \\
\text { weight }(\mathrm{g})\end{array}$ & Spike weight (g) & $\begin{array}{l}1000 \text { kernel } \\
\text { weight }(\mathrm{g})\end{array}$ \\
\hline 1 & 2.93 & 41.15 & 2.81 & 41.00 \\
\hline 2 & 2.72 & 38.56 & 2.72 & 38.56 \\
\hline 3 & 2.82 & 40.75 & 2.45 & 40.26 \\
\hline 4 & 3.98 & 61.00 & 4.00 & 61.08 \\
\hline 5 & 3.56 & 45.00 & 3.80 & 45.20 \\
\hline 6 & 3.80 & 45.30 & 3.62 & 45.10 \\
\hline 7 & 3.90 & 42.10 & 3.90 & 42.10 \\
\hline 8 & 4.78 & 64.20 & 4.31 & 56.23 \\
\hline 9 & 4.40 & 47.90 & 4.14 & 47.30 \\
\hline 10 & 4.84 & 49.30 & 4.84 & 49.30 \\
\hline 11 & 3.66 & 51.90 & 3.30 & 51.90 \\
\hline 12 & 3.51 & 54.30 & 3.58 & 54.36 \\
\hline 13 & 4.90 & 49.60 & 4.20 & 48.12 \\
\hline 14 & 3.57 & 42.10 & 3.57 & 42.10 \\
\hline 15 & 3.70 & 51.80 & 3.27 & 52.00 \\
\hline 16 & 3.50 & 52.00 & 3.50 & 52.20 \\
\hline 17 & 3.70 & 55.20 & 3.70 & 55.20 \\
\hline 18 & 3.82 & 44.52 & 3.82 & 44.52 \\
\hline 19 & 3.78 & 44.00 & 3.78 & 44.00 \\
\hline 20 & 4.62 & 45.90 & 4.22 & 45.00 \\
\hline 21 & 4.81 & 52.10 & 4.31 & 52.00 \\
\hline 22 & 3.90 & 51.50 & 3.33 & 51.80 \\
\hline 23 & 4.66 & 54.40 & 4.62 & 54.14 \\
\hline 24 & 4.00 & 51.33 & 3.50 & 51.00 \\
\hline 25 & 4.18 & 53.20 & 3.68 & 53.00 \\
\hline 26 & 4.62 & 53.80 & 4.60 & 53.18 \\
\hline 27 & 5.00 & 52.72 & 3.88 & 52.22 \\
\hline 28 & 4.04 & 44.00 & 3.65 & 44.10 \\
\hline 29 & 3.10 & 50.40 & 3.90 & 52.40 \\
\hline 30 & 3.50 & 44.40 & 3.85 & 45.12 \\
\hline 31 & 3.42 & 57.56 & 3.12 & 57.06 \\
\hline 32 & 5.00 & 41.20 & 3.92 & 41.00 \\
\hline 33 & 2.98 & 54.30 & 3.00 & 54.90 \\
\hline 34 & 4.10 & 48.70 & 4.11 & 48.22 \\
\hline 35 & 3.50 & 34.00 & 3.18 & 34.01 \\
\hline 36 & 2.60 & 49.20 & 3.12 & 49.11 \\
\hline 37 & 4.40 & 45.70 & 3.33 & 42.15 \\
\hline 38 & 3.30 & 53.80 & 3.52 & 52.95 \\
\hline 39 & 3.62 & 56.70 & 4.00 & 57.00 \\
\hline 40 & 3.64 & 58.70 & 3.50 & 56.70 \\
\hline
\end{tabular}


Table 3: Continued

\begin{tabular}{|c|c|c|c|c|}
\hline 41 & 2.90 & 46.70 & 3.12 & 45.22 \\
\hline 42 & 3.00 & 48.50 & 3.25 & 49.50 \\
\hline 43 & 3.10 & 45.70 & 3.25 & 45.65 \\
\hline 44 & 4.58 & 54.50 & 3.64 & 52.22 \\
\hline 45 & 4.00 & 52.15 & 3.52 & 51.18 \\
\hline 46 & 2.72 & 41.46 & 2.72 & 41.46 \\
\hline 47 & 4.47 & 50.40 & 4.03 & 50.10 \\
\hline 48 & 3.18 & 42.40 & 3.12 & 42.80 \\
\hline 49 & 3.12 & 52.17 & 3.00 & 52.02 \\
\hline 50 & 3.66 & 52.20 & 3.13 & 50.20 \\
\hline 51 & 4.04 & 58.50 & 2.95 & 51.18 \\
\hline 52 & 4.54 & 45.69 & 4.50 & 44.19 \\
\hline 53 & 3.40 & 53.90 & 3.62 & 52.88 \\
\hline 54 & 3.10 & 51.32 & 3.65 & 51.75 \\
\hline 55 & 3.20 & 52.80 & 3.00 & 52.18 \\
\hline 56 & 3.80 & 44.60 & 4.11 & 45.32 \\
\hline 57 & 5.64 & 54.90 & 4.32 & 53.11 \\
\hline 58 & 4.34 & 56.00 & 4.20 & 54.00 \\
\hline 59 & 3.93 & 54.29 & 3.11 & 53.20 \\
\hline 60 & 3.50 & 41.99 & 3.50 & 40.48 \\
\hline 61 & 2.82 & 40.90 & 3.00 & 40.10 \\
\hline 62 & 5.32 & 54.28 & 5.00 & 52.20 \\
\hline 63 & 4.28 & 49.65 & 4.80 & 49.80 \\
\hline 64 & 3.60 & 49.00 & 4.00 & 49.20 \\
\hline 65 & 3.36 & 45.60 & 3.06 & 44.22 \\
\hline 66 & 3.10 & 56.98 & 3.23 & 57.00 \\
\hline 67 & 3.92 & 50.36 & 3.90 & 48.33 \\
\hline 68 & 3.30 & 57.89 & 3.70 & 57.36 \\
\hline 69 & 2.66 & 50.95 & 3.11 & 52.00 \\
\hline 70 & 3.29 & 57.10 & 3.20 & 57.25 \\
\hline 71 & 4.36 & 60.30 & 3.83 & 56.30 \\
\hline 72 & 3.08 & 41.96 & 3.28 & 43.00 \\
\hline 73 & 3.88 & 52.00 & 3.80 & 52.00 \\
\hline 74 & 3.76 & 57.33 & 3.65 & 57.36 \\
\hline 75 & 3.96 & 53.36 & 3.42 & 53.30 \\
\hline 76 & 4.25 & 56.06 & 4.05 & 54.00 \\
\hline 77 & 3.52 & 52.00 & 3.58 & 52.50 \\
\hline 78 & 4.34 & 61.47 & 4.30 & 59.03 \\
\hline 79 & 4.01 & 46.45 & 4.58 & 48.22 \\
\hline 80 & 4.40 & 57.70 & 4.60 & 58.11 \\
\hline 81 & 4.14 & 50.24 & 4.31 & 52.10 \\
\hline 82 & 4.10 & 49.75 & 4.20 & 50.70 \\
\hline 83 & 3.89 & 51.06 & 3.81 & 51.00 \\
\hline 84 & 3.12 & 47.10 & 3.00 & 44.52 \\
\hline
\end{tabular}

Egypt. J. Phytopathol., Vol. 42, No. 2 (2014) 
Table 3: Continued

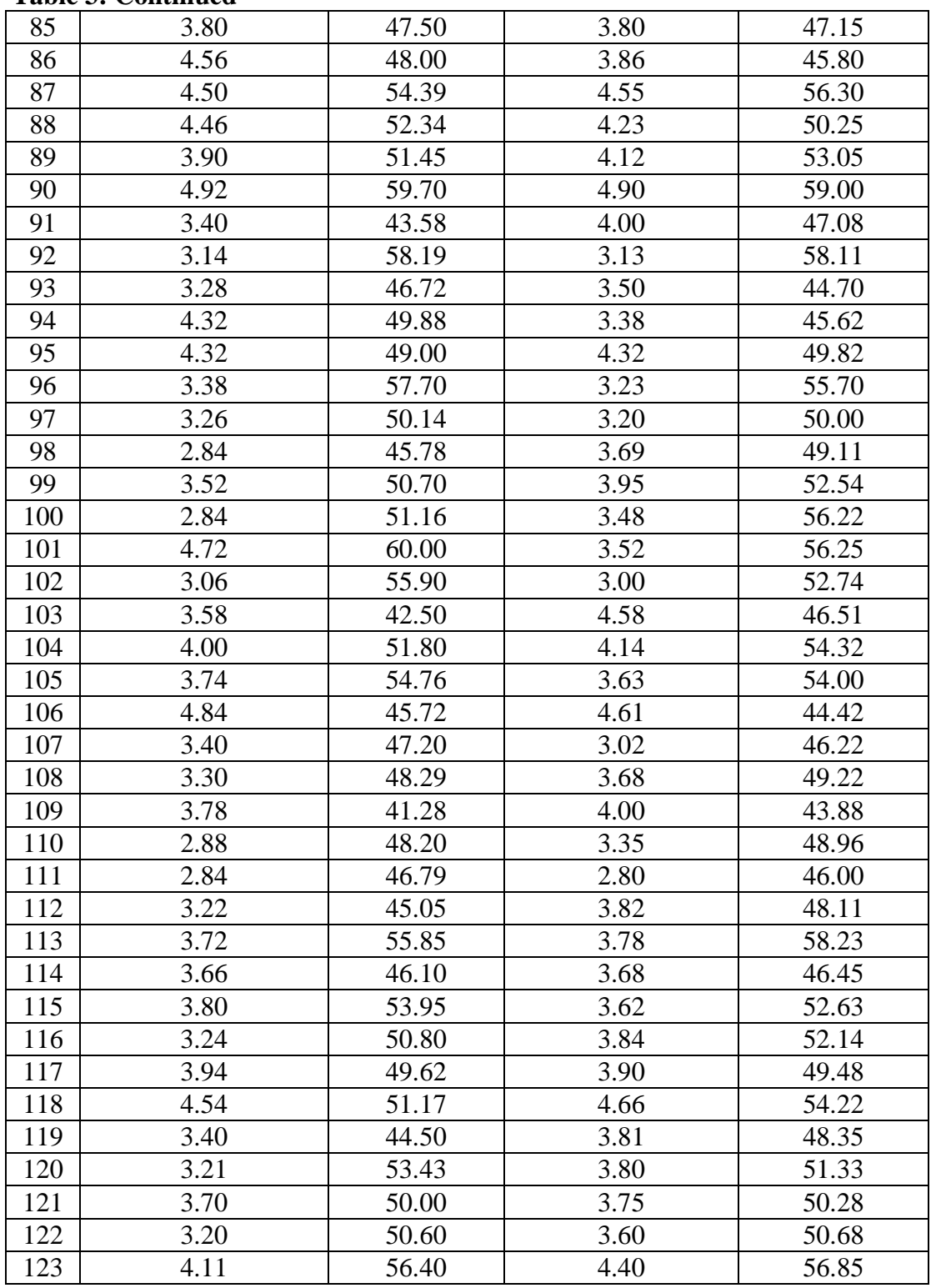




\section{D i s c us s i o n}

Rust diseases of wheat not only reduce the yield but also reduce the grain quality. Using resistant wheat varieties will protect wheat production from disease infection and consequently from yield loss. In this study, 123 wheat genotypes were tested for their resistance to wheat leaf and stem rusts. The tested genotypes were grown at Gemmeiza Research Station during two growing seasons, i.e. 2012/13 and 2013/14. Data on rust incidence were recorded as rust severity (\%), area under disease progress curve (AUDPC) and coefficient of infection (CI) according to the equation adopted by Stubbs et al. (1986).

High yielding and resistant varieties are the main objectives of breeding program in Egypt. In this study, 8 wheat genotypes, i.e. 8, 31, 39, 40, 72, 80, 101 and 123, showed adult plant resistance for both leaf and stem rust diseases during the two successive seasons ranged from 0-10R. Also, these genotypes gave the highest values of yield components. These wheat genotypes were resistant to rust diseases and can be safely used in wheat breeding programs and released as commercial cultivars under Egyptian conditions. Hussain et al. (2010a) found that the score of leaf rust of the wheat variety Mairaj-08 varied from $\operatorname{Tr}$ to $10 \mathrm{MR}$, while it had 0 to Tr for yellow rust during 2005/06 to 2007/08. Also, Mairaj-08 had RRI value of 8-8.9 for leaf rust. Due to better adaptability of the wheat variety Mairaj-08 it has the potential to be approved as a new variety. Hussain et al. (2010b) reported that the rust score of Fareed-06 varied from $5 \mathrm{R}$ to $10 \mathrm{MS}$ for leaf rust and $10 \mathrm{MR} / \mathrm{MS}$ to $10 \mathrm{MS}$ for yellow rust as compared to $70 \mathrm{~S}$ to $100 \mathrm{~S}$ for leaf rust and $50 \mathrm{~S}$ to $90 \mathrm{~S}$ for yellow rust of the check variety, i.e. Morocco. Fareed-06, had RRI value of 8 for leaf and yellow rust. The wheat variety Fareed-06 was approved and released by Punjab Seed Council, Lahore as new variety for cultivation in irrigated areas of Punjab. Hussain et al. (2013) reported that the rust score of the cv. AaS-2011 varied from $10 \mathrm{R}$ to $30 \mathrm{R} / \mathrm{MR}$ for leaf rust and $10 \mathrm{R}$ to $20 \mathrm{MR} / \mathrm{MS}$ for yellow rust as compared to $20 \mathrm{~S}$ to $90 \mathrm{~S}$ for leaf rust and $10 \mathrm{~S}$ to $90 \mathrm{~S}$ for yellow rust of the check variety, i.e. Morocco. AaS-2011 had RRI value of 7.2 to 9 and 7.5 to 9 for leaf and yellow rust, respectively. Cultivar AaS-2011 was approved by Punjab seed Council, Lahore and released as a new variety for general cultivation in hot and drought areas of Punjab. Mahmoud et al. (2013) reported that the rust score of Chakwal-50 varied from $5 \mathrm{MR} / \mathrm{MS}$ to $30 \mathrm{MS}$ for leaf rust and $5 \mathrm{MS}$ to $30 \mathrm{MS}$ for yellow rust. Also, cv. Chakwal-50 had RRI value of 7 to 8.6 and 8 to 8.3 for leaf and yellow rusts, respectively. Cultivar Chakwal-50 has the potential to be approved and released as a new variety. Tariq et al. (2013) stated that the rust score of cv. Dharabi-11 varied from 0 to $5 \mathrm{~S}$ for yellow rust as compared to $80 \mathrm{~S}$ to $90 \mathrm{~S}$ for yellow rust of the check variety Morocco. Cultivar Dharabi-11 had RRI value of 8.8 for yellow rust. Cultivar Dharabi-11 was adapted at different locations, also it has the potential to be approved and released as a new variety. Akhtar et al. (2002) found that seven promising candidate lines, i.e. NR-149, 95C004, 91BT010-5, V-97112, SD1200/14, B96038 and B92044, had desirable/acceptable RRI for leaf rust. So, these lines can be recommended in those areas where rust problem leaf. Rattu et al. (2009) found that out of 29 candidate lines, three lines were found resistant to both leaf and yellow rusts and showed desirable RRI during 2003/04 and 2004/05. 
Worku and Badebo (2012) reported that out of the tested entries, 132 exhibited combined resistances to stem and leaf rust diseases and those were selected for further test in the 2010 off-season. In the subsequent test, 28 lines were identified to have high level of stem rust resistance comparable or better than the resistant checks. The selected durum landraces could be exploited in wheat breeding program.

To increase the wheat production in Egypt, the breeding programs must be selected for yield and its components like the traits studied in this investigation. In these regards data showed that 23 wheat genotypes and 20 wheat genotypes gave the highest values of 1000-kernel weight during the two successive seasons, respectively. On the other hand, 7 and 8 wheat genotypes gave the highest values of spike weight. These results are in harmony with those of Hendawy et al. (2007).

\section{Ref e r e n c es}

Akhtar, M.A.; Ahmad, I.; Mirza, J.; Rattu, A.R.; Ul-Haque, E.; Hakro, A.A. and Jaffery, A.H. 2002. Evaluation of candidate lines against stripe and leaf rusts under National Uniform Wheat and Barley Yield Trial 2000-2001. Asian J. Plant Sci., 4: 450-453.

Anonymous, 2013. Crop production. http://faostat.Fao.org/site/339/olefau/t.aspx

Ashmawy, M.A; El-Orabey, W.M.; Nazim, M. and Shahin, A.A. 2013. Effect of stem rust infection on grain yield and yield components of some wheat cultivars in Egypt. Int. J. Phytopathol., 2(3): 171-178.

Boukhatem, N.; Baret, P.V.; Jacquemin, D. and Mingeot, J.M. 2002. Quantitative trait loci for resistance against Yellow rust in two wheat-derived recombinant inbred line populations. Theor. Appl. Genet., 104: 111-118.

Hendawy, F.A.; Dawwam, H.A. and El-Din, M.M.S. 2007. The detection of the different components of variation in bread wheat (Triticum aestivum L.). Minufiya J. Agric. Res., 32: 1071-1086.

Hussain, M.; Akhtar, L.H.; Rafiq, M.; Aslam, M.Z.; Tariq, A.H.; Aslam, M.; Arshad, M. and Ahmad, S. 2010a. Mairaj-08: New wheat (Triticum aestivum) variety released for general cultivation under normal and late planting in Punjab Province (Pakistan). Int. J. Agric. Biol., 12: 341-347.

Hussain, M.; Hussain, G.; Akhtar, L.H.; Tariq, A.H.; Rafiq, M.; Aslam, M.Z.; Aslam, M.; Arshad, M.; Ahmad, S. and Sahi, S.T. 2010b. New wheat variety "Fareed-06" for irrigated areas of Punjab, Pakistan. Pak. J. Bot., 42: 3258-3297.

Hussain, M.; Rafiq, M.; Akhtar, L.H.; Tariq, A.H.; Ahmad, S.; Aslam, M.Z.; Nadeem, M.A. and Zubair, M. 2013. Release of high yielding wheat variety AaS-2011 resistance to stem rust (Ug-99) in Pakistan. J. Animal Plant Sci., 23: $1115-1124$. 
Kokhmetova, M.A.A.; Rsaliev, S.; Rsaliev, A.; Yessenbekova, G. and Typina, L. 2011. Wheat germplasm screening for stem rust resistance using conventional and molecular techniques. Czech J. Genet. Plant Breed., 47: S146-S154.

Mahmoud, A.; Mian, M.A.; Ihsan, M.; Ijaz, M.; Rabbani, G. and Iqbal, M.S. 2013. Chakwal-50: A high yielding and disease resistant wheat variety for rain fed region. J. Animal Plant Sci., 23: 833-839.

Nazim, M.; El-Shehidi, A.A.; Abdou Y.A. and El-Daoudi, Y.H. 1983. Yield loss caused by leaf rust on four wheat cultivars under epiphytotic levels. Pages: 17-27. In: Proc. $4^{\text {th }}$ Conf. Microbiol., Cairo, Egypt.

Peterson, R.F.; Campbell, A.B. and Hannah A.E. 1948. A diagrammatic scale for estimating rust intensity on leaves and stems of cereals. Can. J. Res., 26: $496-500$.

Rattu, A.R.; Akhtar, M.A.; Fayyaz, M. and Bashir, M. 2007. Screening of wheat against yellow and leaf rusts under NUWYT and NWDSN and wheat rust situation in Pakistan during 2006-2007. Crop Dis. Res. Program, NARC, Islamabad, Pakistan.

Rattu, A.R.; Fayyaz, M.; Ahmad, I.; Akhtar, M.A.; Hakro, A.A. and Khanzada, K.A. 2009. Evaluation of candidate lines against wheat rusts in Pakistan. Pakistan J. Agric. Res., 22: 42-49.

Singh, R.P.; Espino, J.H. and William, H.M. 2005. Genetics and breeding for durable resistance to leaf and stripe rusts in wheat. Turk. J. Agric., 29: 121-127.

Stubbs, R.W.; Prescott, J.M.; Saari, E.E. and Dubin, H.J. 1986. Cereal Disease Methodology Manual. Mexico, CIMMYT. 46pp.

Tariq, M.; Mahmoud, A.; Mian, M.A.; Cheema, N.M.; Sabar, M.; Ihsan, M. and Rehman, A.U. 2013. Dharabi-11: A New high yielding drought and disease tolerant wheat variety. Int. J. Agric. Biol., 15: 701-706.

Tervet, J. and Cassel, RC. 1951. The use of cyclone separation in races identification of cereal rusts. Phytopathology, 41: 282-285.

Worku, D. and Badebo, A. 2012. Valuable sources of resistance in the Ethiopian durum wheat landraces to UG33 and other stem rust races. Int. J. Agron. Plant Prod., 3(6): 191-195.

(Received 17/07/2014; in revised form 16/08/2014) 


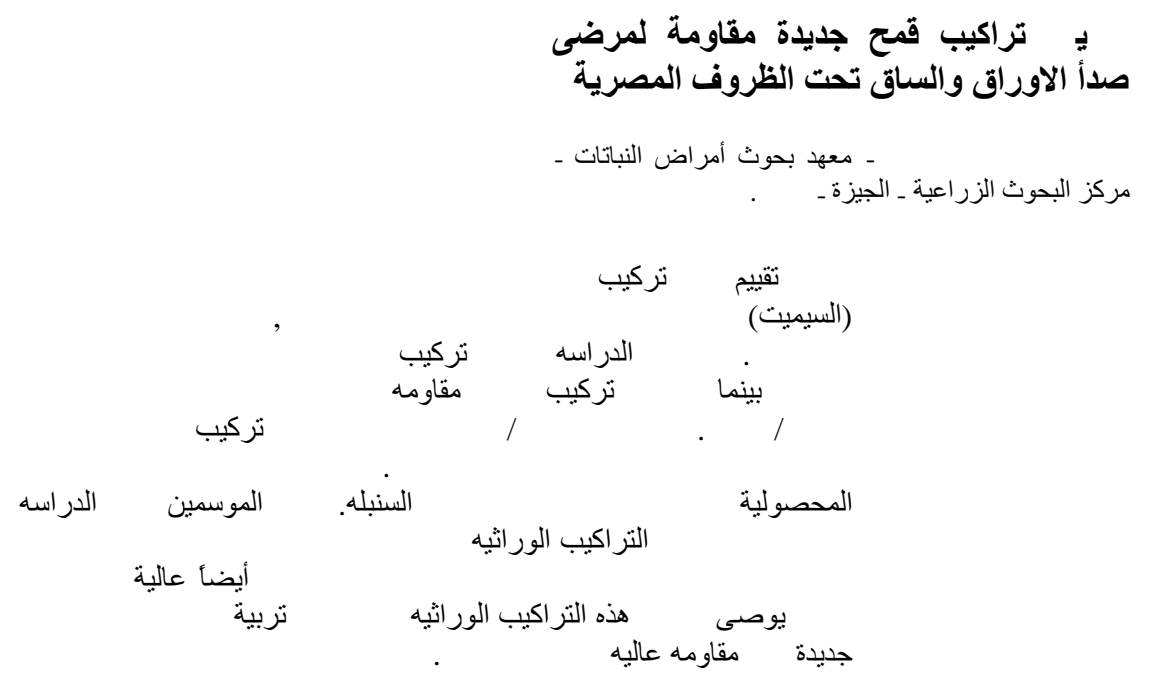

